\title{
Relationship of Ventricular Premature Complexes to Heart Failure (From the Atherosclerosis Risk in Communities [ARIC] Study)
}

\author{
Sunil K Agarwal, MD, PhD ${ }^{a, e}$, Ross J Simpson Jr, MD, PhDa , Pentti Rautaharju, MD, PhD ${ }^{b}$, \\ Alvaro Alonso, MD, $\mathrm{PhD}^{\mathrm{c}}$, Eyal Shahar, MD, $\mathbf{M P H}^{\mathrm{d}}$, Mark Massing, MD, PhD ${ }^{\mathrm{a}}$, Samir Saba, \\ MDe $^{\mathrm{e}}$, and Gerardo Heiss, MD, PhDa \\ aUniversity of North Carolina at Chapel Hill, NC \\ bWake Forest University School of Medicine, Winston-Salem, NC \\ 'University of Minnesota, MN (A.A.) \\ dUniversity of Arizona, Tucson, AZ \\ eUniversity of Pittsburgh Medical Center, PA
}

\begin{abstract}
Analogous to rapid ventricular pacing, frequent ventricular premature complexes (VPCs) may predispose over time to cardiomyopathy and subsequent heart failure (HF). We examined the association of frequent VPCs with HF incidence in a population-based cohort, free of HF and coronary heart disease (CHD) at baseline. At study baseline (1987-89), at least one VPC on a 2minute rhythm ECG strip was seen in 5.5\% (739/13486) of the middle aged (45-64 years old at baseline), white and African-American, men and women of the ARIC cohort. Incident HF was defined as the first appearance of ICD code ' $428 . x$ ' in hospital discharge record or death certificate through 2005. Over an average follow up of 15.6 years, incident HF was seen in $10 \%$ subjects (19.4\% in those with VPCs vs. 9.4\% in those without). The age, race, and gender adjusted hazard ratio (HR) of HF for VPCs was $1.89(95 \% \mathrm{CI}=1.59,2.24)$. After multivariable adjustment for potential confounders, HR (95\% CI) of HF for those with any VPC vs. no VPCs was 1.63 (1.36, 1.96). After additional adjustment for incident $\mathrm{CHD}$ as a time-varying covariate, the $\mathrm{HR}(95 \% \mathrm{CI})$ was $1.71(1.42,2.08)$. Presence of higher frequency of VPCs or complex VPCs had similar rates of HF as compared to single VPC and all were higher than no VPC group. In conclusion, in this large population based cohort, presence of VPCs is associated with incident HF independent of incident CHD.
\end{abstract}

\section{Keywords}

Heart Failure; Ventricular premature complexes; arrhythmias; risk factors; cohort study

\footnotetext{
(C) 2011 Excerpta Medica, Inc. All rights reserved.

Corresponding author: Sunil K. Agarwal, MD, PhD, Address: 137 E. Franklin St., Suite 32, UNC, Chapel Hill, NC, 27514-4145, Phone: sunilagarwal@unc.edu, Phone: (919) 265-4727, Fax: (919) 966-9800.
}

CONFLICTS OF INTEREST None of the author has any conflicts of interest to declare.

Publisher's Disclaimer: This is a PDF file of an unedited manuscript that has been accepted for publication. As a service to our customers we are providing this early version of the manuscript. The manuscript will undergo copyediting, typesetting, and review of the resulting proof before it is published in its final citable form. Please note that during the production process errors may be discovered which could affect the content, and all legal disclaimers that apply to the journal pertain. 


\section{BACKGROUND}

The occurrence of systolic dysfunction and clinical heart failure (HF) with chronic tachyarrhythmia though uncommon, has been well described ${ }^{1}$. Whether frequent Ventricular Premature Complexes (VPCs), a common ${ }^{2}$, yet mostly asymptomatic ectopic arrhythmia, are associated with incident HF is unknown. VPCs are associated with traditional risk factors of coronary heart disease (CHD), a higher incidence of CHD, and cardiovascular death ${ }^{3-4}$. Interestingly, those with frequent VPCs have been shown to have a reduction in ejection fraction ${ }^{5-6}$ and poor diastolic function ${ }^{7}$. Lastly, a few case reports and case series have pointed to the existence of VPC-induced cardiomyopathy, including its reversal with VPC ablation ${ }^{8-11}$. This study examined whether the presence of VPCs on a 2minute ECG recording is associated with incident HF in a large population based cohort, and whether this putative association is present independent of incident CHD.

\section{METHODS}

The Atherosclerosis Risk in Communities (ARIC) Study enrolled 15,792 subjects aged 45-64 years from four U.S. communities: Forsyth County, NC; the city of Jackson, MS; seven northwestern suburbs of Minneapolis, MN; and Washington County, MD (1987-89). African-American residents were over-sampled as part of the area-probability sampling of Forsyth County, NC, while enrollment at the Jackson, MS site was restricted to AfricanAmerican residents. A complete description of the ARIC communities and of the design has been published (http://www.cscc.unc.edu/aric/) ${ }^{12}$. The cohort participated in a baseline visit and three follow-up exams, as well as annual telephone interviews. At study baseline, information about socioeconomic indicators, medical history, family history, cardiovascular risk factors, serum chemistries, ECGs, and medication use was collected.

For the present analysis, participants with prevalent HF (Gothenburg criteria $=3$ or intake of medications (such as digoxin, iv diuretics) for $\mathrm{HF}^{13},(\mathrm{n}=1039){ }^{14}$, or missing information about incident HF were excluded. In addition, participants with missing values for the 12lead ECG or 3-lead 2-minute rhythm strip $(n=74)$ or those with cardiac rhythm disturbances such as Wolf-Parkinson-White syndrome, atrial fibrillation/flutter, wandering atrial pacemaker, supra-ventricular tachycardia, or not-sinus rhythm were excluded ( $\mathrm{n}=$ 283). Participants with missing or invalid values for key covariates $(n=703)$ or self identified their race as neither white nor African-American $(n=48)$ were excluded. Finally, those with prevalent or missing CHD at baseline $(\mathrm{n}=1120)$ were also excluded. Some observations met more than one exclusion criteria. The remaining 13,486 cohort participants were included in this study analyses.

In subset analyses involving adjustment for LVH using Cornell voltage, those with QRS duration $\geq 120 \mathrm{~ms}$ ( $\mathrm{n}=542$ ), were additionally excluded thus leaving 12944 participants for this analysis.

Participants with incident HF were identified using the International Classification of Diseases Code, Ninth Revision (ICD-9) from hospital discharges (428.x), and from death certificates (ICD-9 or ICD-10 codes 428 and I50). Incident HF was defined as the first HF hospitalization with a HF discharge code or HF as the underlying cause of death on a death certificate $(n=68)$. HF cases were identified from three triennial reexaminations through 1998, annual telephone interviews to identify all hospitalizations, as well as through community hospital surveillance for hospitalizations, and searches of National Death Index registry. Cohort follow up for the current analysis ended on 31st December 2005. The validity of ICD code for diagnosis of HF hospitalization has been found to be acceptable in 
the ARIC HF surveillance study - similar to the use of the Framingham criteria for HF (Rosamond et al, poster presented at AHA scientific sessions 2009).

Participants were asked to fast for 12 hours and to refrain from smoking and consuming caffeinated beverages for 4 hours before the examination. A supine 12-lead ECG at rest and a 2-minute 3-lead (V1, II, and V5) rhythm strip were obtained using the MAC PC10 personal cardiogram (Marquette Electronics, Milwaukee, Wisconsin). Electrocardiographic data processing, monitoring, and quality control have been described elsewhere ${ }^{15}$. Rhythm strips were classified 3 times by independent trained coders for total supra-ventricular, ventricular complexes and ventricular runs, bigeminy, trigeminy, and multiform complexes. Adjudication of disagreements was performed by the ECG center principal investigator or the coding supervisor. VPCs and heart rate were determined from the rhythm strip.

The primary exposure was presence of any VPCs on the 2-minute rhythm ECG. VPCs seen on a 2-minute ECG rhythm strip are highly correlated with high frequency VPCs seen on 24 hour recordings ${ }^{16}$. VPCs were classified by the frequency of their occurrence on the 2 minute rhythm strip i.e., single VPC; two or more, and complex. Complex VPCs were defined as presence of consecutive VPCs, multiple morphologies of VPCs, or the presence of short-coupled VPC on the 2-minute ECG.

A parsimonious set of potential confounders for the relation of VPCs to incident HF was chosen based on a strong physiologic relation to $\mathrm{VPCs}^{3}$.

The study protocol manuals on the ARIC website provide detailed information about determination of prevalent CHD (manual 3), hypertension (manual 11), diabetes (manual 10), educational attainment, medication intake history ${ }^{17}$, and Cornell voltage estimated left ventricular $(\mathrm{LV})$ mass $^{18}$ in the ARIC Study ${ }^{19}$. Similarly, the definition of these covariates can be found in the variable dictionary at the ARIC Study website ${ }^{19}$.

Time-to-event for those without incident HF was censored either at death, or at loss to follow up, or on the December 31, 2005, whichever occurred earlier. Rate of HF by race, gender and VPC categories were estimated assuming constant rate over time and a Poisson distribution. Cox regression models were used to estimate the hazard ratio of HF contrasting the presence of any VPC with no VPC. Finally, multivariable Cox regression models were fit including interaction terms between main exposure and age categories, race, gender, diabetes, hypertension, and heart rate (categories at $95^{\text {th }}$ percentile), after adjusting for other potential confounders associated with prevalent VPCs and HF. Relationships by increasing frequency or complexity of VPCs were examined using dummy variables for the categories defined above. Further, to examine the effect of higher frequency of VPCs on HF, we further classified frequencies as $0,1,2$ to 4,5 or greater.

As additional analyses a). Cox model was adjusted for incident CHD as time-varying covariate, and b). adjustment for left ventricular mass using Cornell voltage was done in a subset analysis as this required further exclusion of study participants. The proportional hazard assumption was evaluated both using graphical methods (ln-ln S (t) graphs) and Cox tests and was not invalidated. Multivariable adjusted cumulative HF curves were plotted by presence of VPC using inverse probability weights ${ }^{20}$.

All statistical computations were performed using SAS software version 9.1.3 (SAS Inc., Cary, North Carolina). A p value of $<0.05$ for a two-sided null hypothesis was considered statistically significant for main covariates. A $p$ value of $<0.2$ was considered statistically significant for interaction terms. 


\section{RESULTS}

At least a single VPC was identified in 739 (5.5\%) of the 13,486 study participants using baseline rhythm strip recorded for 2 minutes duration. The differences in the characteristics of the study participants by presence of any VPC and by incident HF status are shown in Table 1.

Incident $\mathrm{HF}$ was seen in $10 \%$ of subjects during an average (SD) follow up period of 15.6 (3.8) years. Cumulative proportion of Incident HF was higher (19.4\%) in those with any VPCs than those without $(9.4 \%)$. The unadjusted rate of HF in individuals with any VPCs was more than twice that of those without (rate $/ 1000$ person year $=13.5$ vs. $6.0, p<0.001$ ).

The age, race, and gender adjusted hazard ratio (HR) of HF for any VPCs was 1.89 (95\% CI $=1.59,2.24)$. After multivariable adjustment for age, gender, race, study center, level of education, blood glucose level, diabetes, systolic blood pressure, intake of antihypertensive medications, LDL-cholesterol, HDL-cholesterol, BMI, smoking status, pack-years of smoking, alcohol intake, heart rate, serum $\mathrm{K}^{+}$, serum $\mathrm{Mg}^{++}$, the $\mathrm{HR}(95 \% \mathrm{CI})$ of $\mathrm{HF}$ among those with any VPC vs. no VPCs was $1.63(1.36,1.96)$. Figure 1 shows the multivariable adjusted cumulative proportion of HF by presence of any VPCs.

The above results did not change appreciably after additional adjustment for 1) left ventricular mass using Cornell voltage (HR 1.59, 95\% CI 1.32, 1.92) or medications such as beta blockers, anti-arrhythmic, and calcium channel blockers. Also, the association existed after adjustment for incident CHD as a time varying covariate (HR 1.71, 95\% CI 1.42, 2.08).

The rate of HF was similar in participants with either 1) a higher frequency or 2) complexity of VPCs when compared to those with single VPC (Figure 2); all of which had higher rates than those without any VPC. However, when examining the effect of higher frequency as single, $2-4,5$ or greater, as compared to none the HR $(95 \% \mathrm{CI})$ were $1.84(1.41,2.41), 1.98$ $(1.49,2.63)$, and $2.16(1.56,3.00)$.

Table 2 shows differences in the association of VPC with HF by strata of selected characteristics. The relationship between VPCs and HF was stronger among whites as compared to African-Americans. Also, the association between VPC with HF was small and statistically non significant in those with diabetes. There was no difference in the association by strata of heart rate.

\section{DISCUSSION}

In this large community-based cohort of white and African-American men and women free of CHD and HF at baseline, we found that VPCs were associated with incident HF. This relationship was independent of several potential confounders including baseline left ventricular mass and incident CHD.

There are several pathways including dys-synchronous ventricular contraction, mechanical bradycardia, tachypacing, and residual confounding due to atherosclerotic burden that can link VPCs with HF. At a basic electrophysiological level, conditions that cause dyssynchrony such as permanent or transient bundle branch blocks have a profound effect on ventricular depolarization and repolarization, affecting action potential durations and leading to adverse remodeling in ventricular dimensions and function. During and following the VPCs, changes in heart rate, blood pressure, and stroke volume may occur as a consequence of changes in ventricular filling, ventricular contractility, and baroreflex activity ${ }^{21}$. In those with frequent PVCs a poorer systolic/diastolic function ${ }^{57}$, a greater deterioration in left ventricular function over time ${ }^{6}$, and a reversal of cardiomyopathy following their ablation in 
selected patients $11,22-23$ has been reported. Also, responders to cardiac resynchronization therapy (CRT) had a lower prevalence of VPCs ${ }^{24}$ - suggesting a role for VPCs in the continuing decline in cardiac function. Lastly, VPCs have been linked to a higher incidence of atrial fibrillation ${ }^{25}$ which can precipitate $\mathrm{HF}^{26}$. Despite several mechanism outlined above, at present the causes of VPC-induced cardiomyopathy remains poorly understood 27 .

Various sources of VPCs have been identified including scar tissue, papillary muscle, Purkinje network system, mitral annulus, and aortic sinus cusps; however, it seems that most of the monomorphic and potentially curable VPCs may originate from the ventricular outflow tract ${ }^{28}$. Many studies suggest the role of VPCs originating from the right ventricular outflow tract in cardiomyopathy $10,22,29$.

Although a higher VPC frequency did show a trend towards slightly increased risk of HF risk in our study, the power was limited; in acute settings, a frequency greater than $10 \%$ has been suggested as a good indicator of response to radiofrequency ablation (RFA) ${ }^{23}$. Similarly, few of those with single VPCs at baseline may actually have complex VPCs if a longer recording is done, this might explain the little difference in the association of complex VPCs with HF as compared to single VPC with HF.

The reasons for lower magnitude of the association between VPCs and HF observed in those with diabetes than those without, is not clear. Higher rates of competing risk of the fatal and non-fatal outcomes we studied among diabetics may account for this. Also, differences in the distribution of unmeasured confounders, differences in the origin/severity of VPCs, or variation in diagnosis/treatment at the sites where African Americans were studied may explain some of these differences.

Strengths of the present study include its large sample size, extensive follow-up, and population-based sampling so that the results can be extrapolated with a greater confidence to the general population. Limitations including poor sensitivity of 2 minute rhythm strip (though highly specific) ${ }^{16}$, and non-availability of serial VPCs evaluation -can cause regression dilution, thus leading to a bias towards null. Another limitation is that our study's definition of HF would likely miss individuals whose HF is successfully managed in outpatient settings, and those who died before their first hospitalization with HF; this would be unlikely given the long follow up in the study and high rate of hospitalizations among patients with HF. Detailed information about HF by echocardiography was not available. However, positive association despite adjustment for incident CHD does support that the relationship is not primarily due to ischemic cardiomyopathy. Unmeasured confounders, although we are not aware of any, may account for some of the association seen in this study. Lastly, though adjustment for manifest incident CHD was done, residual confounding by undiagnosed or silent CHD can't be ruled out. The fact that hazard ratio increased after adjustment of overt CHD is however reassuring that all of the observed risk is not possibly explained by higher rate of CHD in those with VPCs.

\section{Acknowledgments}

FUNDING The Atherosclerosis Risk in Communities Study is a collaborative study supported by National Heart, Lung, and Blood Institute contracts: N01-HC-55015, N01-HC-55016, N01-HC-55018, N01-HC-55019, N01HC-55020, N01-HC-55021, N01-HC-55022, and several other grants.

\section{References}

1. Khasnis A, Jongnarangsin K, Abela G, Veerareddy S, Reddy V, Thakur R. Tachycardia-induced cardiomyopathy: A review of literature. Pacing Clin Electrophysiol. 2005; 28:710-721. [PubMed: 16008809] 
2. Simpson RJ Jr, Cascio WE, Schreiner PJ, Crow RS, Rautaharju PM, Heiss G. Prevalence of premature ventricular contractions in a population of African American and white men and women: the Atherosclerosis Risk in Communities (ARIC) study. Am Heart J. 2002; 143:535-540. [PubMed: 11868062]

3. Massing MW, Simpson RJ Jr, Rautaharju PM, Schreiner PJ, Crow R, Heiss G. Usefulness of ventricular premature complexes to predict coronary heart disease events and mortality (from the Atherosclerosis Risk In Communities cohort). Am J Cardiol. 2006; 98:1609-1612. [PubMed: 17145219]

4. Engel G, Cho S, Ghayoumi A, Yamazaki T, Chun S, Fearon WF, Froelicher VF. Prognostic significance of PVCs and resting heart rate. Ann Noninvasive Electrocardiol. 2007; 12:121-129. [PubMed: 17593180]

5. Sun Y, Blom NA, Yu Y, Ma P, Wang Y, Han X, Swenne CA, van der Wall EE. The influence of premature ventricular contractions on left ventricular function in asymptomatic children without structural heart disease: an echocardiographic evaluation. Int J Cardiovasc Imaging. 2003; 19:295299. [PubMed: 14598897]

6. Niwano S, Wakisaka Y, Niwano H, Fukaya H, Kurokawa S, Kiryu M, Hatakeyama Y, Izumi T. Prognostic significance of frequent premature ventricular contractions originating from the ventricular outflow tract in patients with normal left ventricular function. Heart. 2009; 95:1230 1237. [PubMed: 19429571]

7. Topaloglu S, Aras D, Cagli K, Yildiz A, Cagirci G, Cay S, Gunel EN, Baser K, Baysal E, Boyaci A, Korkmaz S. Evaluation of left ventricular diastolic functions in patients with frequent premature ventricular contractions from right ventricular outflow tract. Heart Vessels. 2007; 22:328-334. [PubMed: 17879025]

8. Chugh SS, Shen WK, Luria DM, Smith HC. First evidence of premature ventricular complexinduced cardiomyopathy: a potentially reversible cause of heart failure. J Cardiovasc Electrophysiol. 2000; 11:328-329. [PubMed: 10749356]

9. Ezzat VA, Liew R, Ward DE. Catheter ablation of premature ventricular contraction-induced cardiomyopathy. Nat Clin Pract Cardiovasc Med. 2008; 5:289-293. [PubMed: 18364706]

10. Takemoto M, Yoshimura H, Ohba Y, Matsumoto Y, Yamamoto U, Mohri M, Yamamoto H, Origuchi H. Radiofrequency catheter ablation of premature ventricular complexes from right ventricular outflow tract improves left ventricular dilation and clinical status in patients without structural heart disease. J Am Coll Cardiol. 2005; 45:1259-1265. [PubMed: 15837259]

11. Bogun F, Crawford T, Reich S, Koelling TM, Armstrong W, Good E, Jongnarangsin K, Marine JE, Chugh A, Pelosi F, Oral H, Morady F. Radiofrequency ablation of frequent, idiopathic premature ventricular complexes: Comparison with a control group without intervention. Heart Rhythm. 2007; 4:863-867. [PubMed: 17599667]

12. The Atherosclerosis Risk in Communities (ARIC) Study: design and objectives. The ARIC investigators. Am J Epidemiol. 1989; 129:687-702. [PubMed: 2646917]

13. Eriksson H, Caidahl K, Larsson B, Ohlson LO, Welin L, Wilhelmsen L, Svardsudd K. Cardiac and pulmonary causes of dyspnoea--validation of a scoring test for clinical-epidemiological use: the Study of Men Born in 1913. Eur Heart J. 1987; 8:1007-1014. [PubMed: 3665952]

14. Loehr LR, Rosamond WD, Chang PP, Folsom AR, Chambless LE. Heart Failure Incidence and Survival (from the Atherosclerosis Risk in Communities Study). Am J Cardiol. 2008; 101:10161022. [PubMed: 18359324]

15. Vitelli LL, Crow RS, Shahar E, Hutchinson RG, Rautaharju PM, Folsom AR. Electrocardiographic findings in a healthy biracial population. Atherosclerosis Risk in Communities (ARIC) Study Investigators. Am J Cardiol. 1998; 81:453-459. [PubMed: 9485136]

16. Evenson KR, Welch VL, Cascio WE, Simpson RJ Jr. Validation of a short rhythm strip compared to ambulatory ECG monitoring for ventricular ectopy. J Clin Epidemiol. 2000; 53:491-497. [PubMed: 10812321]

17. Shahar E, Folsom AR, Romm FJ, Bisgard KM, Metcalf PA, Crum L, McGovern PG, Hutchinson RG, Heiss G. Patterns of aspirin use in middle-aged adults: the Atherosclerosis Risk in Communities (ARIC) Study. Am Heart J. 1996; 131:915-922. [PubMed: 8615310] 
18. Simpson RJ Jr, Cascio WE, Crow RS, Schreiner PJ, Rautaharju PM, Heiss G. Association of ventricular premature complexes with electrocardiographic-estimated left ventricular mass in a population of African-American and white men and women (The Atherosclerosis Risk in Communities. Am J Cardiol. 2001; 87:49-53. [PubMed: 11137833]

19. National Heart Lung and Blood Institute. Atherosclerosis Risk In Communities (ARIC) study.

20. Cole SR, Hernan MA. Adjusted survival curves with inverse probability weights. Comput Methods Programs Biomed. 2004; 75:45-49. [PubMed: 15158046]

21. Malik, M.; Camm, AJ. Dynamic electrocardiography. Elmsford, N.Y.: Blackwell Futura; 2004.

22. Yarlagadda RK, Iwai S, Stein KM, Markowitz SM, Shah BK, Cheung JW, Tan V, Lerman BB, Mittal S. Reversal of cardiomyopathy in patients with repetitive monomorphic ventricular ectopy originating from the right ventricular outflow tract. Circulation. 2005; 112:1092-1097. [PubMed: 16103234]

23. Baman TS, Lange DC, Ilg KJ, Gupta SK, Liu TY, Alguire C, Armstrong W, Good E, Chugh A, Jongnarangsin K, Pelosi F Jr, Crawford T, Ebinger M, Oral H, Morady F, Bogun F. Relationship between burden of premature ventricular complexes and left ventricular function. Heart Rhythm. 2010; 7:865-869. [PubMed: 20348027]

24. Markowitz SM, Lewen JM, Wiggenhorn CJ, Abraham WT, Stein KM, Iwai S, Lerman BB. Relationship of reverse anatomical remodeling and ventricular arrhythmias after cardiac resynchronization. J Cardiovasc Electrophysiol. 2009; 20:293-298. [PubMed: 19175852]

25. Agarwal SK, Heiss G, Rautaharju PM, Shahar E, Massing MW, Simpson RJ Jr. Premature ventricular complexes and the risk of incident stroke: the Atherosclerosis Risk In Communities (ARIC) Study. Stroke. 2010; 41:588-593. [PubMed: 20167922]

26. Nerheim P, Birger-Botkin S, Piracha L, Olshansky B. Heart failure and sudden death in patients with tachycardia-induced cardiomyopathy and recurrent tachycardia. Circulation. 2004; 110:247252. [PubMed: 15226218]

27. Sullivan RM, Olshansky B. Treatment of PVCs post-myocardial infarction: Will we get fooled again? Heart Rhythm. 2009; 6:1550-1551. [PubMed: 19879532]

28. Yamada T, Murakami Y, Yoshida N, Okada T, Toyama J, Yoshida Y, Tsuboi N, Muto M, Inden Y, Hirai M, Murohara T, McElderry HT, Epstein AE, Plumb VJ, Kay GN. Efficacy of electroanatomic mapping in the catheter ablation of premature ventricular contractions originating from the right ventricular outflow tract. J Interv Card Electrophysiol. 2007; 19:187-194. [PubMed: 17891452]

29. Kanei Y, Friedman M, Ogawa N, Hanon S, Lam P, Schweitzer P. Frequent premature ventricular complexes originating from the right ventricular outflow tract are associated with left ventricular dysfunction. Ann Noninvasive Electrocardiol. 2008; 13:81-85. [PubMed: 18234010] 


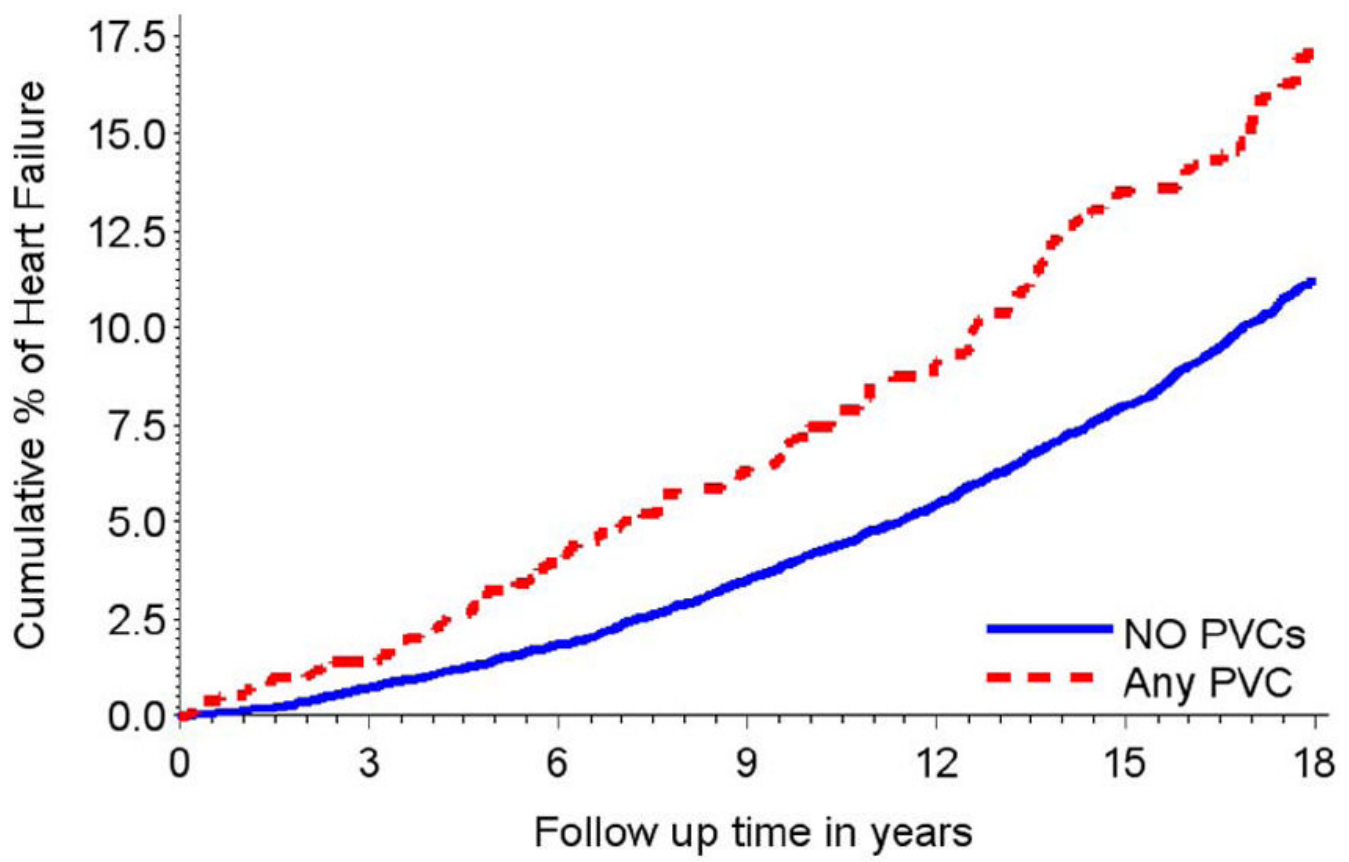

Figure 1.

Multivariable adjusted cumulative heart failure events during follow up by the presence any VPCs (143 HF events among 739) vs. absence (1201 HF events among 12747) events in a 2minute ECG strip among ARIC cohort participants free of heart failure and coronary heart disease at study baseline. (Wilcoxon test $\mathrm{P}<0.001$ ). Model adjusted for age, gender, race, study center, education level, diabetes, systolic blood pressure, hypertension medication intake, LDL and HDL cholesterol, BMI, current smoking, former smoking, pack-years of smoking, amount of ethanol use, heart rate, serum $\mathrm{K}+$, serum $\mathrm{Mg}++$. 


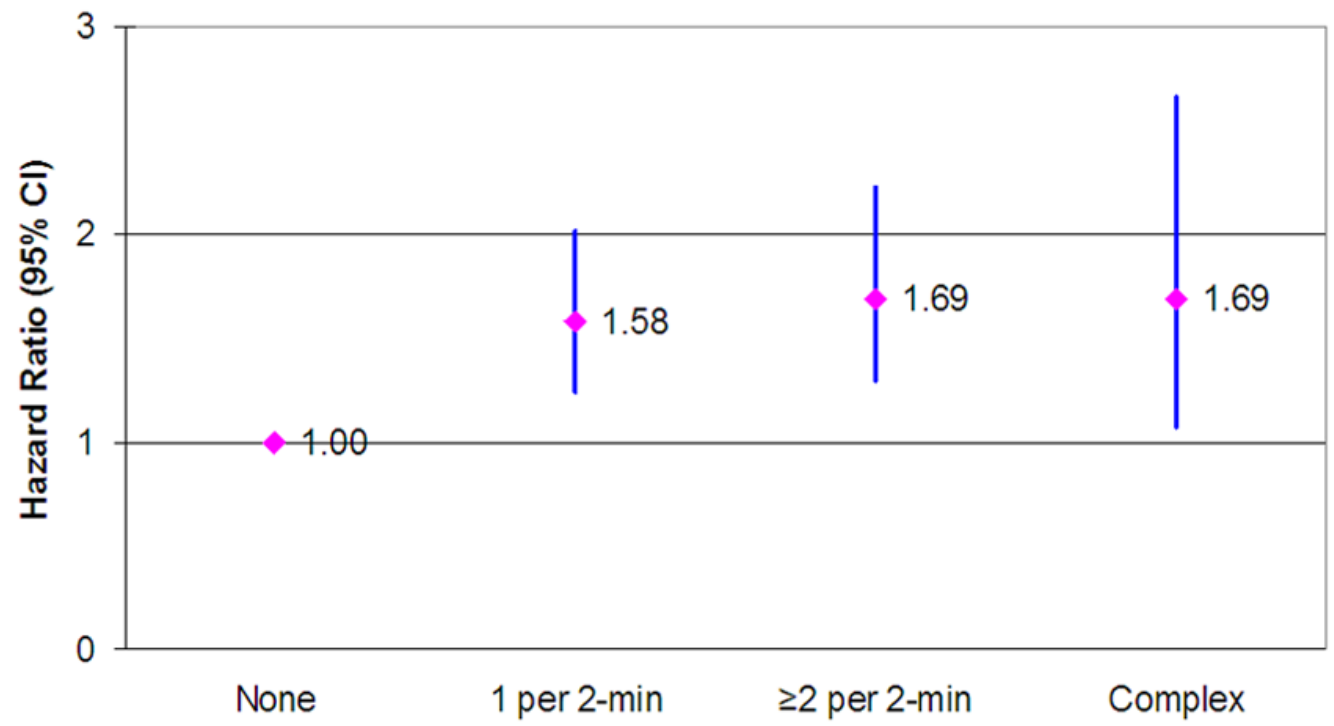

Figure 2.

Hazards ratio of Heart Failure by presence of single $(n=298)$, two-or-more $(n=358)$, and complex $(n=83)$ Ventricular premature complexes (VPCs) as compared to no $(n=12747)$ VPCs in a cohort of middle aged men and women free of CHD at baseline. Cox proportional hazards model adjusted for age, gender, race, study center, education level, diabetes, systolic blood pressure, hypertension medication intake, LDL and HDL cholesterol, BMI, current smoking, former smoking, pack-years of smoking, amount of ethanol use, heart rate, serum $\mathrm{K}^{+}$, serum $\mathrm{Mg}^{++}$. Results from the ARIC study (1987 through 2005). 
Table 1

Characteristics of participants by prevalence of VPC at baseline and occurrence of new-onset Heart Failure during follow up: The Atherosclerosis Risk In Communities Study (1987 to 2005)

\begin{tabular}{|c|c|c|c|c|}
\hline \multirow[t]{2}{*}{ Characteristics ${ }^{*}$} & \multicolumn{2}{|c|}{ Any VPC on 2 minute ECG } & \multicolumn{2}{|c|}{ Heart Failure during follow up } \\
\hline & Yes $(n=739)$ & No $(n=12747)$ & Yes $(n=1344)$ & No $(n=12142)$ \\
\hline Age (in yrs) & $56.0(5.7)$ & $53.8(5.7)$ & $56.5(5.4)$ & $53.7(5.7)$ \\
\hline Women & 49.4 & 56.4 & 50.6 & 56.6 \\
\hline African American & 29.9 & 25.8 & 36.5 & 24.8 \\
\hline \multicolumn{5}{|l|}{$\underline{\text { Education }}$} \\
\hline Less than High School & 27.9 & 21.9 & 39.9 & 20.3 \\
\hline High school but less than college & 41.0 & 41.2 & 36.8 & 41.7 \\
\hline College or more & 31.1 & 36.9 & 23.4 & 38.0 \\
\hline Body Mass Index (kg/m $\left.{ }^{*} \mathrm{~m}\right)$ & $28.2(5.5)$ & $27.4(5.2)$ & $29.6(6.3)$ & $27.2(5.1)$ \\
\hline LDL cholesterol (mg/dl) & $136.5(38.3)$ & $137.1(39.1)$ & $142.6(40.2)$ & $136.5(38.9)$ \\
\hline HDL cholesterol (mg/dl) & $51.4(16.6)$ & $52.6(17.0)$ & $48.1(15.7)$ & $53.0(17.0)$ \\
\hline Potassium (mmol/L) & $4.4(0.5)$ & $4.4(0.5)$ & $4.4(0.5)$ & $4.4(0.5)$ \\
\hline Magnesium (mEq/L) & $1.6(0.2)$ & $1.6(0.2)$ & $1.6(0.2)$ & $1.6(0.2)$ \\
\hline Heart rate (beats/min) & $68.0(10.8)$ & $66.4(10.1)$ & $69.1(11.5)$ & $66.2(9.9)$ \\
\hline Cornell voltage ${ }^{\ddagger}(\mathrm{mV})$ & $1.3(0.6)$ & $1.2(0.5)$ & $1.4(0.6)$ & $1.2(0.5)$ \\
\hline Systolic BP (mm of Hg) & $124.8(19.0)$ & $120.6(18.5)$ & $130.6(21.6)$ & $119.8(17.9)$ \\
\hline Hypertension & $41.5 \%$ & $30.9 \%$ & $54.0 \%$ & $29.0 \%$ \\
\hline Anti-hypertensives & $29.9 \%$ & $21.2 \%$ & $39.6 \%$ & $19.7 \%$ \\
\hline Glucose & $109.5(40.0)$ & $106.9(36.8)$ & $130.5(69.2)$ & $104.4(30.4)$ \\
\hline Diabetes & $11.5 \%$ & $8.1 \%$ & $25.7 \%$ & $6.3 \%$ \\
\hline Current smoking & $25.2 \%$ & $25.9 \%$ & $37.6 \%$ & $24.5 \%$ \\
\hline Former Smoking & $33.6 \%$ & $31.3 \%$ & $31.0 \%$ & $31.5 \%$ \\
\hline Pack-years of smoking & $17.3(23.1)$ & $14.9(20.6)$ & $22.7(26.1)$ & $14.2(19.9)$ \\
\hline Alcohol intake (gms/week) & $45.9(118.8)$ & $42.1(92.1)$ & $38.7(91.5)$ & $42.7(94.0)$ \\
\hline Any $\mathrm{VPCs}^{\dagger}$ & $100 \%$ & 0 & $10.6 \%$ & $4.9 \%$ \\
\hline Single & $40.3 \%$ & 0 & $3.9 \%$ & $2.0 \%$ \\
\hline More than one VPC & $48.4 \%$ & 0 & $5.3 \%$ & $2.4 \%$ \\
\hline Complex VPCs & $11.2 \%$ & 0 & $1.4 \%$ & $0.5 \%$ \\
\hline
\end{tabular}

* Characteristics expressed as percentage of column or means with standard deviation

${ }^{\dagger}$ VPC coded by investigators using 2-minute (3-lead ECG strips) at baseline

Cornell voltage was calculated in those with QRS $<120 \mathrm{~ms}$, with non-missing values, and not having prevalent CHD.

Complex VPCs were defined by the presence of consecutive, multi-morphic, or short coupled ( $<5$ percentile). 
Table 2

Multivariable adjusted Cox regression model for Heart Failure*: the Atherosclerosis Risk In Communities Study (1987 through 2005)

\begin{tabular}{|ccccc|}
\hline Stratum & Hazard ratio $^{\dagger}$ & $\mathbf{9 5 \%}$ CI & Interaction P value \\
\hline Overall & 1.63 & 1.37 & 1.95 & \\
Age $<55$ years & 1.80 & 1.30 & 2.51 & 0.29 \\
Age $>=55$ years & 1.60 & 1.30 & 1.96 & \\
Female & 1.44 & 1.10 & 1.87 & 0.02 \\
Male & 1.83 & 1.45 & 2.32 & \\
African American & 1.44 & 1.07 & 1.93 & 0.13 \\
White & 1.77 & 1.42 & 2.20 & \\
Diabetes & 1.26 & 0.88 & 1.82 & 0.01 \\
No Diabetes & 1.83 & 1.50 & 2.24 & \\
Hypertension & 1.62 & 1.29 & 2.03 & 0.46 \\
No Hypertension & 1.72 & 1.30 & 2.28 & \\
Heart rate $<85$ & 1.64 & 1.36 & 1.98 & 0.87 \\
Heart rate $>=85$ & 1.62 & 0.99 & 2.67 & \\
\hline
\end{tabular}

* Incident heart failure cases from study enrollment (1987-89) through 2005

${ }^{\dagger}$ Estimates are hazard ratio for presence of any VPC in 2-minute (3-lead ECG strips) at study baseline from multivariable models in each stratum. With the exception of corresponding row variable, each model was adjusted for age, gender, race, study center, education level, diabetes, systolic blood pressure, hypertension medication intake, LDL and HDL cholesterol, BMI, current smoking, former smoking, pack-years of smoking, amount of ethanol use, heart rate, serum $\mathrm{K}^{+}$, serum $\mathrm{Mg}^{++}$. Heart rate of 85 corresponds to the $95^{\text {th }}$ percentile for ARIC study cohort at baseline visit.

FInteraction term includes the exposure variable (presence of any VPCs) and the corresponding row variable; a p value corresponding to this product term is labeled as interaction $\mathrm{p}$ value and a value $<0.05$ suggests strong statistical interaction. 